\title{
Zur Kasuistik der Hypotrichosis congenita familiaris.
}

Von

Dr. Th. Baer, Frankfurt am Main.

Es unterliegt wohl nach den bisher vorliegenden Veröffentlichungen keinem Zweifel mehr, daß es sich bei der Hypotrichosis congenita um eine relativ seltene Bildungsanomalie handelt, die auch bei mehreren Mitgliedern derselben Familie beobachtet wurde.

Die Ausführungen von Pinkus, welche das Wesen der Anomalie am besten erklären, seien hier wiedergegeben. „Nicht der Haarausfall, sondern der mangelnde Haarwuchs ist der pathologische Vorgang; er setzt genau in dem Momente ein, wo bei normaler Haarbildung an Stelle der fötalen Lanugo eine stärkere Behaarung des Kopfes beginnt. Durch diesen Anschluß an den normalen Vorgang des Ausfalles fötaler Kopflanugo wird es sehr wahrscheinlich, daß es sich hier nicht um eine Krankheit, sondern um eine fehlerhafte Anlage der bleibenden Haarschicht, um eine Mißbildung, handelt."

Und weiter: "Hier also, nach Beendigung des fötalen Haarausfalls, beginnt erst die Abweichung, von der Norm. Die Hypotrichosis liegt vollkommen im Gebiete des bleibenden Haarwuchses."

Sie hat also zur Voraussetzung, daß die fötale Haaranlage ausgebildet war, daß aber der Nachwuchs des bleibenden Haarkleides unterblieben ist.

Es sind nun in der Literatur eine Reihe von Fällen niedergelegt, die aus zwei Gründen einer strengen Kritik nicht Stand halten können, und zwar deshalb, weil

1. eine Kombination mit anderen Dermatosen bestand,

2. aus der Anamnese nicht nachgewiesen werden konnte, daß der Haarmangel ein angeborener war. 
Ad 1. Unter dem Bilde des angeborenen Haarmangels sind Fälle beschrieben, bei welchen anderweitige Hauterkrankungen vorlagen. Diese haben streng genommen mit einer kongenitalen Anomalie nichts $z u$ tun, da die Alopecie auch als Folgeerscheinung der Hauterkrankung angesehen werden kann. So lag bei dem Fall von Luce's Xerodermie vor. Bei dem Audryschen Fall Keratose, bei dem Fall von Jones und Aitkens werden atrophische Vorgänge beschrieben, ferner deutet Kaposi den Fall Zieglers als lichen pilaris.

Ad 2. Kraus, der die einzelnen Fälle auf ihre anamnestische Zuverlässigkeit hin prüfte, erwähnt als Typen der Hypotrichosis congenita nur die Fälle von Fordyce, Abraham, Pinkus und seinen Fall.

Die beiden Voraussetzungen, völliges Feblen jeglicher anderer Hanterkrankung, anamnestischer Nachweis der fötalen Lanugo und späterer Ausfall derselben, mangelnder bzw. fast völlig unterbliebener Nachwuchs, treffen bei den im folgenden zu beschreibenden Fällen zu, die ich gelegentlich einer, zu Ehren der Anwesenheit Neissers stattgehabten Versammlung südwestdeutscher Dermatologen bereits vorgestellt habe.

Die Eltern der Patienten geben an, daß sie immer gesund waren und niemals über Erscheinungen von seiten der Haut oder der Haare zu klagen hatten. Die Untersuchung ergibt, daß die Haut und deren Anhangsgebilde absolut normal entwickelt sind. Aus der Ehe gingen zehn Kinder hervor, von diesen hatten 6 normale Haut- und Haarbeschaffenheit, während \pm völlig haarlos waren, bzw, sind.

1. Der älteste Sohn, jetzt 22 Jahre alt (Fall 1), kam vollständig haarlos zur Welt. Die Eltern bemerkten während seines ganzen Lebens auch nicht ein Haar, mit Ausnahme der Augen. brauen und Lider. Die übrige geistige und körperliche Entwicklung war vollständig normal.

2. Normal entwickelt, starb mit $16 \mathrm{Jahren}$ an Kehlkopfschwindsucht.

3. Madchen, 18 Jahre (Fall 2). Dasselbe brachte nach der bestimmten Angabe beider Eltern ziemlich reichlich Haare und zum Teil ganz lange Haare mit auf die Welt, die aber nach 14 Tagen fast alle a usfielen. Etwa 10-15 Kopfhaare sollen noch während des ersten Lebensjahres bestanden haben, dann fielen auch diese vollständig aus. Seit der Zeit sei kein Haar mehr aufgetreten.

4. Vollständig normal behaart, starb mit 15 Jahren an Lungenphthise. 5., 6. und 7. Normal behaart, gesund. 
8. Mädchen (Fall 3) kam mit reichlichen Haaren auf die Welt, hat dieselben mit dreiviertel Jahren dagegen völig verloren. Blieb haarlos bis zu seinem, im fünften Lebensjahre erfolgten Tode.

9. Mädchen, 8 Jahre (Fall 4), kam mit langen, aber spärlichen Haaren auf die Welt und verlor dieselben nach 14 Tagen, blieb seit dieser Zeit haarlos.

10. 4 Jahre alt, am Leben, normaler Haarwuchs.

Status praesens. Die drei Fälle haben so vieles gemeinsam, daß sich eine gesonderte Besprechung jedes einzelnen nicht verlohnt. Es sind 3 kräftig gebaute, blühend aussehende Individuen, die auch psychisch einen durchaus normalen Eindruck machen. Die ganze Haut des Körpers ist haarlos, mit Ausnahme der Augenbrauen und (bei Fall 1) der Schurrbartgegend. Ich zählte in Fall 1 auf der inneren Hälfte der linken Augenbraue 9, auf der entsprechenden anderen 10 Haare. Schnurrbarthaare waren 2 vorhanden; bei Fall 2 auf der linken Augenbraue 13, auf der rechten 20 Haare; bei Fall 4 rechts 7, links 8 Haare. Die untersuchten Haare waren markhaltig. Von Flaumhaaren ist bei keinem der 3 Fälle etwas wahrzunehmen gewesen.

Nirgends läßt sich auf der Haut eine Anomalie nachweisen. Û̉berall sind die Follikel deutlich ausgebildet, nirgends pathologische Veränderungen. Die Haut ist normal pigmentiert, zeigt normale Faltbarkeit, Schweißsekretion bei allen Fällen vorhanden, Sensibilität überall intakt. Zähne und Nägel völlig normal.

Es lassen sich also aus unserer Krankengeschichte folgende Punkte mit Sicherheit feststellen.

1. Es war die primäre Anlage in drei Fällen nach den absolut zuverlässigen Angaben der beiden Eltern unzweifelhaft vorhanden. (Fall $1 \mathrm{kam}$ zwar haarlos zur Welt, doch ist in Analogie mit den 3 anderen Fällen anzunehmen, daß hier wohl die primäre Anlage bestanden hat, aber ante partum abgestoben wurde.)

2. Es hat sich eine bleibende, neue Haarbildung nicht eingestellt.

3. Es sind klinisch irgendwelche andere Hautveränderungen nicht nachzuweisen. Eine Biopsie wurde leider nicht gestattet.

Wir sind also berechtigt unseren Fall als den Typus einer angeborenen, familiären Hypotrichose zu bezeichnen.

Der familiäre Charakter der Hypotrichosis congenita wurde bisher außerordentlich selten beobachtet ( $\mathrm{Kraus}$ ).

Die Ätiologie ist völlig dunkel; es werden Syphilis, Lichen pilaris, Ichthyosis, nervöse Einflüsse als Ursachen angegeben.

Areh. f. Dermat. u. Syph. Bd. LXXXIV. 
Hievon ist bei unseren Fällen, wie schon oben erwähnt, nichts nachzuweisen. Ebensowenig scheint eine Disposition eines Geschlechtes zu bestehen. Die von uns daraufhin geprüften Fälle ergeben ein fast gleichmäßiges Befallensein beider Geschlechter. (S. auch Marcuse.) $O b$ in unserem Falle der Tatsache, daß die Eltern verwandt (Geschwisterkinder) waren, eine Bedeutung beizumessen ist, sei dahingestellt.

Zur Klassifizierung der mit Haarausfall einhergehenden Erkrankungen bzw. Anomalien sind mehrere Schemata (Brocq, Besnier, Bettmann) angegeben, ron denen uns das Bettmannsche als das geeignetste erscheint. Dasselbe betont nicht die Art, sondern das zeitliche Einsetzen der Schädigung. keime.

a) Die Störung betrifft den Fötus vor Anlage der Haar-

b) Die Störung wirkt auf den Fötus während der Ausbildung der Primärhaare.

c) Die Störung betrifft den fötalen Haarwechsel.

Mögliche Folgen:

1. Der Haarwechsel unterbleibt.

2. Der Haarwechsel verringert sich.

Die Unterbringung unserer Fälle in dieses Schema bietet nun insofern keine Schwierigkeiten, als eine Störung des fötalen Haarwechsels außer Zweifel steht. Trotz der fast völligen Haarlosigkeit können wir — und zwar wegen der vorhandenen, wenn auch außerordentlich spärlichen Sekundärhaare an den Augenbrauen bzw. Schnurrbart - nur von einer Verzögerung des Haarwechsels sprechen.

Noch ein Wort zur Nomenklatur. Es ist den Autoren nur beizupflichten, wenn sie die Bezeichnung "Alopecia" nur für die Fälle reserviert wissen wollen, bei welchen es sich um einen pathologischen Ausfall schon vorhanden gewesener Haare handelt. Dagegen sollten alle Fälle, bei welchen die Abweichung von der Norm nicht im Haarausfall, sondern in dem $\mathrm{ihm}$ folgenden mangelnden Nachwuchs (Pinkus) besteht, als "Hypotrichosis" bezeichnet werden.

\section{Literatur.}

Neisser. Handbuch der prakt. Medizin (Ebstein). Bd. III. 2. Teil.

Ziegler. Arch. f. Dermatologie and Syphilis. Bd. XXXIX.

Pinkus. Arch. f. Dermatologie und Syphilis. Bd. L.

Bettmann. Arch. f. Dermatologie und Syphilis. Bd. LV.

Kraus. Arch. f. Dermatologie und Syphilis. Bd. LXVI.

Marcuse. Hautkrankheiten und Sexualität. 1907.

Eschner. Ref. Arch. f. Dermatologie and Syphilis. Bd. LXXVIII.

La pratique dermatologique. Bd. I.

Besnier-Dyon. Maladie de la peau. 1891. 